equipments for the work, and if additional transport is required for them it must be provided." Mr. Watson Cheyne is evidently very strongly impressed with the dangers and the horrors which of necessity accompany all attempts to remove seriously wounded soldiers for any distance after an engagement, and he thinks that the ideal plan would be to arrange for the field hospitals to be left on the field of battle until it was safe for them to be transported to the base. He thinks some arrangement might be come to among the nations in order that this might be done. In the meantime what is wanted is better arrangements to prevent sepsis in the first instance, proper supplies of lotions and boiled water, proper illumination (for the operative work after a battle has to be done by night), and an increase in the personnel.

1 Birmingham Medical Review, November, 1901.

\section{DERANGEMENTS OF THE THYROID} GLAND.

THe effects produced by the removal of the thyroid gland are so marked, and the results of treating myxedema by thyroid extract have been so surprising, that it is not unnatural that its use in other conditions in which no such striking results have been obtained should have led to a general feeling that the therapeutic uses of thyroid extract have but a narrow range; one limited, in fact, to the relief of such symptoms as are known to be producible by the removal of the gland. Still, one meets here and there with cases in which this extract is decidedly useful, and anyone who can so group them and give such a clinical picture of them as shall guide us to the sort of case in which we may expect benefit from the administration of thyroid extract does us a good service. Hence much interest attaches to a paper read by Dr. John Perry ${ }^{1}$ at the October meeting of the Harvard Medical Society of New York on the "Functional Disorders of the Thyroid Gland." In this paper he shows that a certain group of cases which have been confused with rheumatism are in some way connected with disorder of the thyroid and are capable of being relieved by the administration of thyroid extract. In the cases described five symptoms were usually present, of which pain in the joints was the most prominent. The others were a feeble and rapid heart; a tendency to headache, not localised but general ; menorrhagia, in women; and usually the fact that the development of the symptoms came on after a shock or fright. An important point was that most of the cases observed had in consequence of the joint pain been regarded as cases of rheumatism or gout. The joints were not red and usually were not very tender, but there was frequently a pallid swelling with slight tenderness, resembling the condition which develops in the so-called neural arthropathy described by Charcot. In one of Dr. Perry's patients, an unmarried woman who was gradually drifting into a helpless condition of invalidism, in addition to pallor of the face, the eyes were drawn together and the pinched expression ard compressed lips indicated an intensely neurotic condition. This was particularly noticeable during her painful attacks. These were localised in the knee, which was puffy and tender but not reddened. It may be noted that these attacks were not associated with flatulence nor with gastric disturbance, and did not simulate migraine. The day after an attack she would be up, feeling reasonably well, although without appetite and physically depressed. In this case the condition was attributed to the shock of a railway accident, and many of her symptoms were undoubtedly mental. But in this as in other cases the supra-sternal notch was more or less obliterated, and here was to be found the only physical sign that seemed to provide a possible rational indication for treatment. It is here that the interest of the cases largely lies, viz., in the fact that they improved under the use of thyroid extract. As was pointed out in the discussion the administration of thyroid must often be purely empirical. It certainly will not do to restrict its use to those cases alone in which we know, as we know of myxedema, that it will do good. It is only by trial that the extent of its utilities can be discovered, and if by further trial it should be shown that Dr. Perry is correct in attributing to thyroid extract the power of curing the group of symptoms which he has described, we shall have gained a step. In the meantime as an indication for empirical treatment the association of these symptoms with a filling up. of the suprasternal notch is a thing to remember.

$$
1 \text { New York Med. News, Nov. } 16 .
$$

\section{SYPHILITIC BRAIN DISEASE.}

Is the course of a lecture at the Queen's Square Hospital, delivered on October 22nd, Sir William Gowers drew attention to certain points of mucb interest in regard to prognosis and treatment in cases of syphilitic disease of the brain. The diagnosis may be right as to the syphilitic nature of the disease, even though as between tumours and arterial. disease it may be wrong. In the latter condition the greatest care is necessary in giving a prognosis. There are certain conditions when, for example, a syphilitic arteritis implicates the whole circumferenceof the vessel, in which restoration is impossible. Whatever may be the precise way in which treatment acts upon syphilitic growths we know that their ultimate fate is to be changed into cicatricical fibrous tissue which will shrink and contract, and thus it is that when an artery is involved in its. whole circumference the final result must be an interference with the circulation, depending as to its. degree partly on the amount of the disease, partly on the possibility of collateral circulation becoming. established. Hence, however confident we may be as to our power of influencing a syphilitic growth, we can by no means be sure of the persistence of the lumen of a syphilitic artery. Moreover, there is always the possibility of the formation of clot in such vessels, and when an artery is so closed its cavity cannot be restored. All this has to be thought of in regard to prognosis, for with our knowledge as to the control which remedies have over syphilitic disease there is no doubt a tendency to allow the nature of the constitutional malady to influence the prognosis favourably without sufficient consideration being given to the particular process to which the symptoms are directly due. When the blood supply is interfered with by an obstruction in an artery the effects, to which the symptoms are due, are of a purely secondary and simple nature, and the fact of syphilis existing cannot influence the prognosis. Coming now to the treatment and consuder 
ing the use of iodide in cases of cerebral syphilis, although this drug must be given, the treatment must be carefully adjusted to the patient's state. One must not forget the power with which iodide is credited of promoting the coagulation of the blood, lest by giving over large doses one might facilitate the formation of a clot in a narrowed vessel. A dose of 15 grains three times a day is large enough to influence such disease if the patient has not been taking it before, and is as much as it is well to give under the circumstances. The lessened susceptibility to a drug, and the lessened therapeutical activity of a drug in those who are accustomed to its use are matters of much interest. To obtain the full influence of iodide for example, and the same is true of mercury, the system must not be accustomed to its presence. It may happen that a moderate dose of iodide, such as 10 grains three times a day, may quickly cause the disappearance of all symptoms of a gumma in the brain, and yet while this same dose is being continued another gumma may develop. Evidently the organısms by which syphilitic disease is produced may become so accustomed to the remedies by which they are usually controlled as to develop in spite of them. Hence the importance of treatment being energetic but also intermittent.

\section{THE FREQUENCY OF GALL STONES.}

THere are certain conditions which are much more frequently discovered by the pathologist than by the physician, and among such is the existence of gall stones. Many a patient suffers for years from more or less ill-defined ailments which are put down to chronic dyspepsia, constipation, billiousness, or what not, and the real origin of which is never discovered until perhaps the pathologist finds a gall-bladder full of calculi. Even then it may be impossible to speak with certainty as to the relationship between the gall stones and the maladies, but, at least, it may be stated that, in many cases, if it could have been known during life that these stones were present, they would almost certainly have been regarded as the probable explanation of the symptoms exhibited by the patient If we did but recognise the unreliability of the generally accepted symptoms of gall stones and the frequency with which these concretions exist without showing any of the recognised signs of their presence, we should probably be much more ready than we are to think of gall stones in all cases of abdominal trouble associated with painful indigestion. Dr. Fiedler ${ }^{1}$ has lately stated that out of 93,000 patients examined, gall stones were noted during life in only 133, i.e., in 0.14 per cent., while at autopsies gall stones are found in fully 10 per cent. of the bodies examined if a careful examination of the biliary system be made. The difference between the frequency of the antemortem and the post-mortem discovery of gall stones is very striking, and shows in how many cases this possible cause of many troubles remains undiscovered and unsuspected. Not that we would suggest that the gall stones are the cause of all the abdominal symptoms which occur in cases where they exist, for, indeed, there is much reason for believing that even before a gall stone forms there has been some preceding catarrh or disorder of the biliary passages. Nor can one say, as some might perhaps suggest, that these figures show in how large a number of cases treatment ought to have been surgical rather than medical, for even after removal of gall stones recurrence takes place in a not inconsiderable proportion of the cases. What these figures really show is that biliary catarrh, even in its slighter forms, is a more serious affair and more common complaint than some have imagined, and that in cases in which there is reason to believe that it may have occurred, treatment should not be confined to the mere relief of the symptoms which have produced distress, but should be so long continued and should take such a direction as to restore the healthy action of the liver and thus prevent the formation of biliary calculi.

$$
1 \text { Münch. Med. Woch., Oct. } 25 .
$$

\section{IRRIGATION OF THE URETHRA.}

Dr. Louis Broter ${ }^{1}$ evidently exaggerates greatly when he says that the irrigation method of treating. acute gonorrhœa " is one of the greatest blessings. that human thought and observation have furnished to mankind." Still there may be some good in the treatment, notwithstanding the style in which it is set forth by its advocate. Dr. Broter first insists. upon the necessity of obtaining complete asespis of all the nozzles and other instruments employed, and he recommends the use of certain appliances which are doubtless of considerable advantage in carrying out any such treatment, for example the wearing of an indiarubber apron by the patient, as he stands before the surgeon during the applications, the apron having a hole for the penis in the middle of it. The solution employed is one of permanganate of potassium. 1 in 4,000 increased up to 1 in 1,000 as the disease advances. Of this solution a quart is to be used at each application. Its temperature should be at least $100^{\circ} \mathrm{F}$. and may be increased up to $112.120^{\circ} \mathrm{F}$. as the patient becomes accustomed to it. The stream is directed into the meatus with gradual increase of force. "Should it be desired to wash out the posterior urethra or the bladder the nozzle must be brought into close proximity to the meatus, at the same time telling the patient to attempt to urinate. This last act, as will be readily understood, loosens the cut-off muscles and the solution gains an entrance. This is continued until an entire quart of the solution is used." After the irrigation three drachms of a 2 per cent. solution of protargol are injected through an ordinary urethral syringe. This solution is retained, if possible, for five or ten minutes, when it is allowed to flow away. The number of irrigations required varies. For the first two or three weeks they may have to be given twice a day, and then, when the discharge has changed from purulent to serous, they may be given once a day and followed by injections of zinc or lead, with or without bismuth, instead of the protargol injections. Even after the urine is clear and the discharge and gonococci have disappeared, the irrigations are to be continued once a week for another month. It is evident then that unless gonorrhoa is a very much more serious affair in New York than it is with us, this treatment does not offer any great advantage in regard to the time or the care required. Nor does it appear that even Dr. Broter trusts exclusively to. irrigation, for not only does he employ the abovementioned injections but he gives alkalies and diuretics, such as bicarbonate of soda and acetate of potash, while he finds methylene blue and boracic 